\title{
History of Set Theory and Its Extensions in the Context of Soft Computing
}

\author{
Sarjerao Nimse ${ }^{1}$ and Pawan Lingras ${ }^{2}$ \\ 1 Vice-Chancellor \\ Swami Ramanand Teerth Marathwada University \\ Vishnupuri, Nanded, 431 606, Maharashtra, India \\ dr.sbnimse@rediffmail.com \\ 2 Department of Mathematics and Computing Science, Saint Mary's University \\ Halifax, Nova Scotia, B3H 3C3, Canada \\ pawan@cs.smu.ca
}

\begin{abstract}
The arithmetization programs of mathematicians in later half of nineteenth century showed that it is necessary to rebuild analysis. Riemanns attempt to find necessary and sufficient conditions for representation of a function by its Fourier series led to the recognization of different types of infinite sets. This fact motivated Georg Cantor to develop unified theory of sets. In fact Cantors interest in set theory stemmed from his researches on trigonometric series in general and fourier series in particular. Nowadays, theory of sets and functions forms the foundation upon which the structure of modern mathematics is built.

Lotfi A. Zadeh, in 1965, published his seminal paper on Fuzzy Sets. The membership in a fuzzy set is not a matter of affirmation or denial, but rather a matter of degree. The significance of Zadehs work is that it led to study of uncertainty from most general point of view, restricting classical probability theory to study certain types of uncertainty only. In the process, Aristotelian two valued logic is generalized to Fuzzy Logic, which is an infinite valued logic. During last four decades researchers realized the tremendous scope of fuzzy sets and fuzzy logic in Soft Computing. Neural networks, Neuro-Fuzzy Modeling, Neuro-Fuzzy Control, Fuzzy Databases and Information retrieval Systems, Fuzzy Decision Making are some of the important areas of research persued globally.

Z. Pawlak, in the year 1982, proposed Rough set theory, as a new mathematical approach to imperfect knowledge. Rough set theory is complementary to and can also be often used jointly with other approaches such as statistical methods, neural networks, genetic algorithms, fuzzy sets etc. Rough set approach is found important for Artificial Intelligence and Cognitive Sciences including data mining.

In this paper, we propose comparative advantages of fuzzy sets and rough sets in the context of Soft Computing.
\end{abstract}

